\title{
Vollzeit- und Teilzeiterwerbstätigkeit im Familienzyklus US-amerikanischer Frauen - Eine Längsschnittanalyse
}

\section{Sonja Drobnič \\ Immo Wittig}

Universität Bremen, Sonderforschungsbereich 186, Postfach 330 440, D-28334 Bremen

\begin{abstract}
Zusammenfassung: In diesem Beitrag werden der langfristige Wandel im Erwerbsverhalten amerikanischer Frauen und die Bedeutung von Veränderungen in der familiären Konstellation für die Erwerbspartizipation erörtert. Zunächst skizzieren wir, wie sich dic Frauenerwerbstätigkeit in den USA entwickelt hat und zeigen auf, weshalb diese Entwicklung nur mithilfe einer Längsschnittanalyse zu verstehen ist. Anschließend werden Übergänge zwischen Beschäftigungssystem und Nichterwerbstätigkeit anhand von Erwerbsbiographien des „National Survey of Families and Households" untersucht. Es ergibt sich, daß die gestiegene Frauenerwerbstätigkeit kaum durch einen Verzicht auf Erwerbsunterbrechungen im Lcbenszyklus zu erklären, sondern auf veränderte Muster des Wiedereintritts in das Beschäftigungssystem zurückzuführen ist. Hierbei erweisen sich Vollzeit- und Teilzeitarbeit als strukturell verschiedene Formen der weiblichen Erwerbsbeteiligung. Die Aufnahme von Teilzeitarbeit entpuppt sich vor allem als ein Phänomen jüngerer Geburtskohorten, während gleichzeitig seit den 70er Jahren Vollzeiterwerbstätigkeit für Frauen aller Jahrgänge und Altersstufen typischer geworden ist. Die weitere Analyse zeigt für die USA deutlich schwächere Effekte familiärer Ereignisse auf weibliche Erwerbsbeteiligung als für Westdeutschland berichtet wurden. Besonders erwähnenswert ist, daß in den USA dic Aufnahme von Teilzcitarbcit nicht von familiären Bedingungen abhängt.
\end{abstract}

\section{Einleitung}

Fragen zur Teilzeiterwerbstätigkeit haben in Deutschland in den vergangenen Jahren eine Schwerpunktverlagerung erfahren. Ursprünglich gehörte der Ruf nach mehr Teilzeitarbeitsplätzen zur Standardforderung, um Frauen eine Erwerbstätigkeit in Zeiten der Kindererziehung zu ermöglichen. Angesichts der Ausweitung, die Teilzeitarbeit nicht nur in Deutschland, sondern in den mejsten westeuropäischen Ländern erfahren hat, könnte man dieser Politik einen ziemlichen Erfolg bescheinigen. Gegenwärtig ist vor dem Hintergrund der Diskussion über die Zukunft der Arbeitsgesellschaft die Umverteilung von Arbeit durch Teilzeitarbeit in den Vordergrund des Interesses gerückt.

Sowohl im Hinblick auf die Umverteilung von Arbeit, als auch was den Zusammenhang zwischen Familie und Teilzeitarbeit anbelangt, hilft das Verstehen längerfristiger Entwicklungen auf dem Arbeitsmarkt, die Grenzen eines auf der Schaffung von Teilzeitarbeitsplätzen basierenden Lösungsansatzes zu erkennen. Welche Bedingungen der Angebotsseite des Arbeitsmarktes begünstigen die Aufnahme von Vollzeit- bzw. Teilzeiterwerbstätigkeit? Ist reduzierte Erwerbsarbeit an bestimmte Etappen im Karriereverlauf gekoppelt? Welche Rolle spielen hierbei familiäre Ereignisse im Lebenslauf von Individuen, und welche Funktion hat speziell Teilzeitarbeit bei der Integration von Frauen in den Arbeitsmarkt? In welcher Weise haben sich die veränderte Struktur des Beschäftigungssystems und der Konjunkturverlauf auf die beiden Beschäftigungsformen ausgewirkt? Eine Längsschnittanalyse der Erwerbsbiographien von US-Amerikanerinnen soll wichtige strukturelle Gründe für die Aufnahme bzw. für das Verlassen von Teilzeit- und Vollzeiterwerbstätigkeit offenlegen. In diesem Zusammenhang soll demonstriert werden, daß eine Längsschnittanalyse mit Individualdaten geradezu die conditio sine qua non zum Verständnis sozialen Wandels im Erwerbsverhalten ist.

Da sich bei Frauenerwerbstätigkeit und Teilzeitarbeit insbesondere weitaus größere Unterschiede zwischen einzelnen Ländern feststellen lassen, als bei der (Vollzeit-)Erwerbstätigkeit von Männern. ist ein internationaler Vergleich besonders gewinnbringend. Zwar sind auch in den USA die Teilzeitarbeitsplätze mehrheitlich mit Frauen besetzt. doch sie arbeiten in einem weitaus höherem Maße vollzeit als in Westdeutschland. Sowohl das hohe Ausmaß weiblicher Teilzeitbeschäftigung als auch deren Expansion sind spezifisch für West- und Nordeuropa (vgl. auch Blossfeld 1995; Drobnič 1995). Die langfristige Betrachtung des Erwerbsverhaltens zeigt, daß sich die Arbeitsmarktintegra- 
tion von Frauen in den USA vergleichsweise stark über Vollzeiterwerbstätigkeit realisiert hat statt wie in Westdeutschland über Teilzeitarbeit. Hierfür ist in erster Linie eine unterschiedliche Ausgestaltung des Gegensatzes zwischen Erwerbstätigkeit und Reproduktionsarbeit verantwortlich. Am Beispiel USA wird offenkundig. daß der in Westdeutschland enge Zusammenhang zwischen weiblicher Teilzeiterwerbstätigkeit und familiären Verpflichtungen (vgl. Blossfeld/Rohwer 1995) keine universelle Gültigkeit beanspruchen kann. Dies weckt erhebliche Zweifel an der immer noch weit verbreiteten Meinung, der Wandel der Frauenerwerbstätigkeit verlaufe in westlichen Industrieländern nach einem im wesentlichen gleichen Muster.

\section{Frauenerwerbstätigkeit und Teilzeitarbeit in den USA: Langfristige Entwicklungs- linien}

In den meisten westeuropäischen Ländern stellt die starke Zunahme von Teilzeitarbeit eine der überragenden strukturellen Veränderungen des Arbeitsmarktes seit den fünfziger Jahren dar, was weder in einschlägigen wissenschaftlichen Publikationen noch in der Öffentlichkeit in dieser Form wahrgenommen wurde. Teilzeitarbeit heute ist nicht nur fast ausnahmslos Frauenarbeit geblieben; bemerkenswert ist vor allem, daß die Frauenerwerbsquote in vielen Ländern zum größten Teil durch die Zunahme von Teilzeitarbeit gesticgen ist. In Großbritannien beispielsweise stieg von Anfang der fünfziger bis Ende der achtziger Jahre im wesentlichen die Anzahl teilzeitbeschäftigter Frauen, die Zahl der insgesamt von Frauen geleisteten Arbeitsstunden änderte sich hingegen kaum. Besonders stark wuchs die Zahl der Teilzeitarbeitsplätze in Westeuropa während der Hochkonjunktur der sechziger und siebziger Jahre; in den Vereinigten Staaten weitete sich im Gegensatz zu dieser Entwicklung besonders die Vollzeitbeschäftigung bei Frauen aus. ${ }^{1}$ Hakim (1993)

\footnotetext{
1 Zur Entwicklung in Großbritannien siehe Becchey/Perkins (1987). Hakim (1993), Burchell et al. (1993); für Schweden siehe Sundström (1991); für Westdeutschland siche Quack (1993). Blossfeld/Rohwer (1995); Ellingsæter (1992) vergleicht Dänemark, Deutschland, Norwegen und Großbritannien. Nach OECD-Daten für 1990/1991 betrug der Anteil teilzeitbeschäftigter Frauen an allen erwerbstätigen Frauen: $62,2 \%$ in den Niederlanden. $47.6 \%$ in Norwegen, $43.2 \%$ in Großbritannien. $40.5 \%$ in Schweden. $38,4 \%$ in Dänemark, $34,3 \%$ in Japan. $33,8 \%$ in Deutsch-
}

hat unter dem beziehungsreichen Titel „.The $M y t h$ of Rising Female Employment" (Hervorhebung von den Autoren) eindrucksvoll gezeigt. daß es ohne Differenzierung zwischen Vollzeit- und Teilzeitarbeit zu einer Unterschätzung der geschlechtsspezifischen Struktur des Arbeitsmarktes kommt, oder anders gewendet: zu einer illusionär anmutenden Überschätzung der Frauenemanzipation.

Für die Vereinigten Staaten ist im Laufe des 20. Jahrhunderts und besonders seit den vierziger Jahren ein zunächst schwaches und sich dann beschleunigendes Wachstum der Frauenerwerbsquote zu verzeichnen (Abbildung 1). Die erwerbstätige Frau vor 1940 kann als jung, arm und alleinstehend charakterisiert werden, sie arbeitete typischerweise nur bis zu ihrer Heirat. Schätzungen

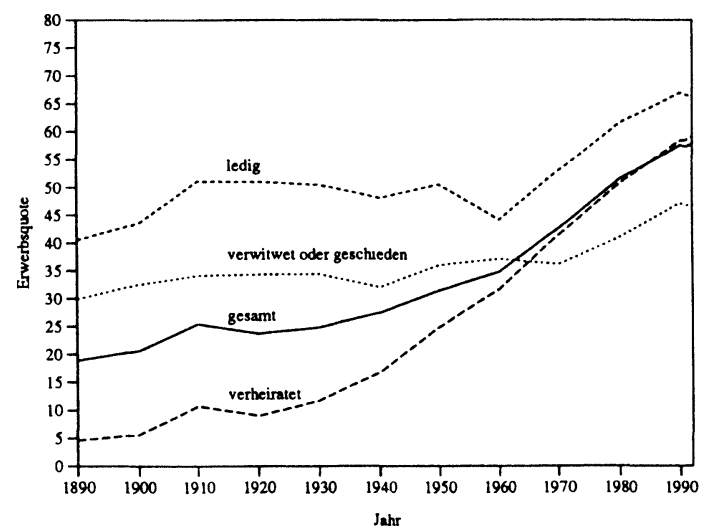

Abb. 1 Anteil der Erwerbspersonen (civilian labor force) bei US-amerikanischen Frauen.

Frauen ab 16 Jahre (bzw. ab 14 Jahre bis 1960) in Privathaushalten.

Quellen: für 1890-1930: Wandersee (1981), nach Tabelle 4.1 .

für 1940-1992: Statistical Abstract of the United States, diverse Ausgaben (basierend auf Current Population Survey).

land, $25,6 \%$ in den Vereinigten Staaten, $23,5 \%$ in Frankreich. $20.2 \%$ in Österreich. $11.2 \%$ in Spanien und $9.6 \%$ in Italien. Die Vereinigten Staaten liegen somit international gesehen im unteren Bereich. Bei teilzeitarbeitenden Männern hingegen nimmt die USA eine Spitzenposition ein. Die entsprechenden Quoten liegen bei $16,7 \%$ für die Niederlande, $10,5 \%$ für die USA, $10,4 \%$ für Dänemark, $10,1 \%$ für Japan, $9,1 \%$ für Norwegen. $7,5 \%$ für Schweden, $5.3 \%$ für Großbritannien, $3.4 \%$ für Frankreich, $2.6 \%$ für Deutschland, $2,4 \%$ für Italien, $1,6 \%$ für Österreich und $1.5 \%$ für Spanien. 
zufolge waren von den knapp vier Millionen im Zensus von 1890 als erwerbstätig registrierten Frauen und Mädchen nur rund 500000 verheiratet (Hesse 1979). Die hierfür verantwortliche Praxis sogenannter „marriage bars" - expliziten Regeln, verheiratete Frauen nicht einzustellen bzw. Frauen nach ihrer Heirat zu kündigen - war weit verbreitet und für einen großen Teil frauentypischer Berufe bis in die fünfziger Jahre hinein wirksam.

Gründe für Veränderungen im Erwerbsverhalten amerikanischer Frauen sind weniger in einer allmählichen Änderurig von Einstellungen und Rollenerwartungen zu suchen, sondern die historische Zäsur des 2. Weltkriegs brachte einen entscheidenden Durchbruch. In der Zeit von 1940 bis 1945 strömten Frauen aus allen sozialen Schichten auf den Arbeitsmarkt, die Anzahl erwerbstätiger Frauen stieg in nur wenigen Jahren um etwa fünfeinhalb Millionen. Nach Kriegsende wurden mit der Rückkehr der Männer auf zivile Arbeitsplätze Frauen zwar einem massiven Druck ausgesetzt, das Erwerbssystem wieder zu verlassen (Hesse 1979), die Zahl erwerbstätiger Frauen fiel jedoch nur kurzzeitig etwas ab, der Trend zugunsten weiblicher Erwerbstätigkeit blieb ungebrochen und hat sich seither nicht umgekehrt. Nach Oppenheimer (1973) bewirkte in der Nachkriegsperiode das Wechselspiel zwischen einer demographisch bedingten Verknappung des Arbeitskräfteangebots und der Expansion traditionell weiblich dominierter Tätigkeiten eine rasche Integration von Frauen in den Arbeitsmarkt. Weil das Angebot der traditionsgemäß bevorzugten jungen unverheirateten Frauen rückläufig war, ließ sich der steigende $\mathrm{Ar}$ beitskräftebedarf nicht mehr decken, ohne vermehrt ältere und verheiratete Frauen einzustellen. Darüber hinaus gab es zunehmend mehr junge Mütter, die aufgrund ihrer besseren und aktuelle-

Tabelle 1: Erwerbstätigkeit in den USA (employed persons) für die Jahre 1968-1992.

\begin{tabular}{|c|c|c|c|c|c|c|c|}
\hline \multirow[t]{2}{*}{ Jahr } & \multirow{2}{*}{$\begin{array}{l}\text { Teilzeit- } \\
\text { quote } \\
\text { insgesamt }\end{array}$} & \multicolumn{2}{|c|}{ Männer } & \multicolumn{2}{|c|}{ Frauen } & \multicolumn{2}{|c|}{ Frauenanteil (in \%) } \\
\hline & & $\begin{array}{l}\text { insgesamt } \\
\text { (in 1000) }\end{array}$ & $\begin{array}{l}\text { Teilzeit- } \\
\text { quote }\end{array}$ & $\begin{array}{l}\text { insgesamt } \\
\text { (in 1000) }\end{array}$ & $\begin{array}{l}\text { Teilzeit- } \\
\text { quote }\end{array}$ & $\begin{array}{l}\text { Vollzeit- } \\
\text { besc }\end{array}$ & $\begin{array}{l}\text { Teilzeit- } \\
\text { ftigte }\end{array}$ \\
\hline 1968 & 14,0 & 48114 & 7,8 & 27807 & 24,9 & 32,0 & 65,0 \\
\hline 1969 & 14,5 & 48818 & 8,2 & 29084 & 25,1 & 32,7 & 64,6 \\
\hline 1970 & 15,2 & 48990 & 8,5 & 29688 & 26,1 & 32,9 & 65,1 \\
\hline 1971 & 15,6 & 49390 & 8,8 & 29976 & 26,8 & 32,6 & 64,8 \\
\hline 1972 & 15,7 & 50896 & 8,9 & 31257 & 26,9 & 33,0 & 65,0 \\
\hline 1973 & 15,6 & 52349 & 8,6 & 32715 & 26,8 & 33,4 & 66,0 \\
\hline 1974 & 15,8 & 53024 & 8,8 & 33769 & 26,8 & 33,8 & 66,1 \\
\hline 1975 & 16,6 & 51858 & 9,4 & 33989 & 27,6 & 34,4 & 65,9 \\
\hline 1976 & 16,6 & 53138 & 9,4 & 35613 & 27,5 & 34,9 & 66,3 \\
\hline 1977 & 16,7 & 54729 & 9,5 & 37289 & 27,4 & 35,3 & 66,4 \\
\hline 1978 & 16,5 & 56479 & 9,2 & 39569 & 26,9 & 36,1 & 67,2 \\
\hline 1979 & 16,4 & 57607 & 9,0 & 41217 & 26,7 & 36,6 & 68,0 \\
\hline 1980 & 16,8 & 57186 & 9,6 & 42117 & 26,8 & 37,4 & 67,3 \\
\hline 1981 & 17,1 & 57397 & 9,6 & 43000 & 27,1 & 37,6 & 68,0 \\
\hline 1982 & 18,2 & 56271 & 10,6 & 43256 & 28,1 & 38,2 & 67,2 \\
\hline 1983 & 18,4 & 56787 & 10,8 & 44047 & 28,1 & 38,5 & 66,8 \\
\hline 1984 & 17,6 & 59091 & 10,2 & 45915 & 27,1 & 38,7 & 67,4 \\
\hline 1985 & 17,3 & 59891 & 10,1 & 47259 & 26,6 & 39,2 & 67,6 \\
\hline 1986 & 17,4 & 60892 & 10,2 & 48706 & 26,4 & 39,6 & 67,4 \\
\hline 1987 & 17,4 & 62107 & 10,2 & 50334 & 26,1 & 40,0 & 67,4 \\
\hline 1988 & 17,3 & 63273 & 10,2 & 51696 & 25,7 & 40,3 & 67,3 \\
\hline 1989 & 17,0 & 64315 & 10,0 & 53028 & 25,5 & 40,6 & 67,8 \\
\hline 1990 & 16,9 & 64434 & 10,0 & 53479 & 25,2 & 40,8 & 67,6 \\
\hline 1991 & 17,4 & 63593 & 10,5 & 53283 & 25,6 & 41,0 & 67,2 \\
\hline 1992 & 17,5 & 63804 & 10,8 & 53793 & 25,4 & 41,4 & 66,4 \\
\hline
\end{tabular}

Erwerbstätige ab 16 Jahre (bzw. ab 14 Jahre bis 1960) in Privathaushalten.

Teilzeitquote: Anteil Teilzeitbeschäftigter an Erwerbstätigen (in \%)

Quellen: für 1968-1988: ILO (1989), nach Tabelle 19.

für 1989-1992: Statistical Abstract of the United States, diverse Ausgaben (basierend auf Current Population Survey). 
Tabelle 2: Enwerbstätige und Teilzeitquote nach Alter und Geschlecht in den Jahren 1969/1979/1989.

\begin{tabular}{|c|c|c|c|c|c|c|}
\hline \multirow[b]{2}{*}{ Population } & \multicolumn{2}{|c|}{1969} & \multicolumn{2}{|c|}{1979} & \multicolumn{2}{|l|}{1989} \\
\hline & $\begin{array}{l}\text { Anteil an } \\
\text { den Erwerbs- } \\
\text { tätigen }\end{array}$ & $\begin{array}{l}\text { Teilzeit- } \\
\text { quote }\end{array}$ & $\begin{array}{l}\text { Anteil an } \\
\text { den Erwerbs- } \\
\text { tätigen }\end{array}$ & $\begin{array}{l}\text { Teilzeit- } \\
\text { quote }\end{array}$ & $\begin{array}{l}\text { Anteil an } \\
\text { den Erwerbs- } \\
\text { tätigen }\end{array}$ & $\begin{array}{l}\text { Teilzeit- } \\
\text { quote }\end{array}$ \\
\hline $\begin{array}{l}\text { insgesamt } \\
\text { im Alter 16-21 }\end{array}$ & 12,8 & 40,6 & 14,0 & 41,7 & 10,3 & 46,3 \\
\hline $\begin{array}{l}\text { Frauen } \\
\text { im Alter 22-44 }\end{array}$ & 17,3 & 22,7 & 23,1 & 22,5 & 27,7 & 21,9 \\
\hline $\begin{array}{l}\text { Frauen } \\
\text { im Alter 45-65 }\end{array}$ & 13,2 & 22,5 & 11,3 & 24,4 & 11,6 & 23,8 \\
\hline $\begin{array}{l}\text { Männer } \\
\text { im Alter 22-64 }\end{array}$ & 53,2 & 3,7 & 48,9 & 4,8 & 47,8 & 6,7 \\
\hline $\begin{array}{l}\text { insgesamt } \\
\text { im Alter über } 65\end{array}$ & 3,5 & 41,1 & 2,7 & 52,9 & 2,6 & 52,4 \\
\hline insgesamt & 100.0 & 15,5 & 100,0 & 17,6 & 100,0 & 18,1 \\
\hline
\end{tabular}

\section{Ohne Landwirtschaft}

Teilzeitquote: Anteil Teilzeitbeschäftigter in der jeweiligen Population in\%.

Quelle: Tilly (1991): 11, Tabelle 1 (eigene Berechnungen von Tilly auf Basis der BLS-Publikation „Employment and Earnings" für die Jahre 1970, 1980 und 1990).

ren Ausbildung eine starke Position auf dem expandierenden Arbeitsmarkt hatten. Wie Oppenheimer (1974) ebenfalls verdeutlicht hat, war der Eintritt dieser Gruppe in den Arbeitsmarkt auch das Resultat früher Heirat: viele junge Paare waren nicht in der Lage, vom Verdienst des Ehemannes allein zu leben.

Gegenwärtig ist von den Erwerbstätigen in den USA fast die Hälfte (46 Prozent) weiblich. 1992 arbeitete in den Vereinigten Staaten jede vierte erwerbstätige Frau und jeder zehnte Mann teilzeit (definiert mit weniger als 35 Wochenarbeitsstunden). 41 Prozent der Vollzeitbeschäftigten und zwei Drittel der ungefähr 20 Millionen Teilzeitbeschäftigten waren weiblich (Tabelle 1). Im Zeitraum von 1968 bis 1992 hat sich in den USA die Anzahl erwerbstätiger Frauen beinahe verdoppelt. Der Frauenanteil der Vollzeitbeschäftigten ist beträchtlich angestiegen, der Frauenanteil der Teilzeitbeschäftigten ist jedoch ebenso wie die Teilzeitquote der Frauen bemerkenswert konstant geblieben.

Ein erster Erklärungsansatz für Teilzeitarbeit liegt darin, daß sie bei beiden Geschlechtern mit Phasen im Lebenszyklus zu Beginn und gegen Ende der beruflichen Laufbahn in Verbindung gebracht werden kann, also für einige Personen den Einstieg in den Arbeitsmarkt vorbereitet und später dann in die Pensionierung überleitet. Tabelle 2 zeigt für 1969, 1979 und 1989 die hohe und im Zeit- verlauf steigende Teilzeitquote jüngerer (Alter 1621 Jahre) wie älterer (über 65 Jahre) Personen. Diese Daten lassen auch die möglicherweise überraschende Tendenz erkennen, daß Frauen im kindererziehenden Alter (22-44 Jahre) ihre Erwerbsbeteiligung deutlich gesteigert haben, der Anteil Teilzeitbeschäftigter in dieser Altersgruppe jedoch nicht angestiegen ist.

\section{Theoretischer Rahmen und Hypothesen über weibliche Erwerbsbeteiligung}

Nach der Humankapitaltheorie begünstigt ein erhöhtes Bildungsniveau die Arbeitsmarktintegration. Höhere Bildung steigert den Marktwert und damit die Opportunitätskosten einer Nichterwerbstätigkeit (Becker 1981). Die Höherqualifizierung der jüngeren Geburtskohorten - im Jahre 1952 war ein Drittel der amerikanischen Universitätsabsolventen weiblich, bis Mitte der achtziger Jahre stieg dieser Anteil auf über die Hälfte (Jacobs 1989) - kann deshalb als direkter Indikator für das weibliche Erwerbspotential gewertet werden und läßt in der Kohortenfolge eine Zunahme der Erwerbsbeteiligung erwarten (vgl. Goldin 1990). Als weiterer Grund für die Zunahme der Frauenerwerbstätigkeit ist oft der Funktionswandel der Institution Familie genannt worden. Hohe Scheidungsraten und nicht-eheliche Geburten haben die Zahl alleinerziehender Mütter anwach- 
sen lassen (Bumpass 1990; DaVanzo/Rahman 1993). Es ist unwahrscheinlicher geworden, durch Heirat eine lebenslange ökonomische Sicherheit zu erreichen. Gerade junge Frauen dürften aus dem Wandel der Familie vermehrt den Wunsch nach wirtschaftlicher Unabhängigkeit ableiten. Auch für verheiratete Frauen in den USA ist eine Erwerbstätigkeit notwendiger geworden, weil ein Alleinernährer aufgrund realer Einkommensverluste seit den 70er Jahren immer weniger zur Versorgung der Familie in der Lage ist (vgl. Gerson 1985). Die heute in allen westlichen Industrieländern niedrige Geburtenrate schließlich hat die zur Kindererziehung notwendige Zeit reduziert und auch der Einzug der Technik in den Haushalt erlaubt es Frauen, mehr Zeit im Arbeitsmarkt zu verbringen.

Welches individuelle Erwerbsverhalten sich indessen hinter der gestiegenen Frauenerwerbstätigkeit verbirgt, ist umstritten. Einige Autoren betonen, weibliche Erwerbsbeteiligung in den USA sei nach wie vor eng mit familiären Verpflichtungen verknüpft (Moen/Smith 1986). Anderen Studien kann man entnehmen, daß Frauen in der heutigen Zeit für kontinuierliche Arbeitsmarktpartizipation optieren (Mellor/Parks 1988; Smith/Ward 1989). Unumstritten ist, daß in modernen Gesellschaften in aller Regel Frauen die Hauptverantwortung für Kindererziehung und Hausarbeit tragen - selbst in Ehen, in denen beide Partner erwerbstätig sind (Presser 1994). Da Zeitkontingente begrenzt sind, gewinnt in diesem Rollenkonflikt zwischen Erwerbs- und Hausarbeit die Länge der Arbeitszeit eine zentrale Bedeutung: Teilzeitarbeit wird zur typischen „Option“, besonders dann, wenn jüngere Kinder zu betreuen sind (Bernhardt 1993).

Ansätzen aus der Ökonomie zufolge bestimmt die Zuständigkeit für den häuslichen Bereich nicht nur maßgeblich die Verweildauer von Frauen im Erwerbssystem, sondern ist auch für die geschlechtsspezifische Berufswahl und für die Erwerbsorientierung mitverantwortlich (Mincer/Polachek 1974; Mincer/Ofek 1982; Polachek 1981). Zwar werden in der Humankapitaltheorie Aussagen über Erwerbsarbeit generell gemacht, doch lassen sich auch Vermutungen über die Funktion von Teilzeitarbeit ableiten. Die strenge Spezialisierung auf Produktions- bzw. Reproduktionsarbeit verliert durch Faktoren wie Geburtenrückgang, Anstieg von Ehescheidungen, attraktivere Verdienstmöglichkeiten für Frauen ihre ursprünglichen Vorteile. Wenn jedoch mehrere Mitglieder eines Haushaltes die gleichen relativen Vorteile besitzen, investiert unter rationalen Akteuren höch- stens ein Mitglied dieses Haushaltes Zeit und Energie in beide Bereiche - Arbeitsmarkt und Haushalt (Becker 1981: 18). Die Abwendung von traditionellen Rollen liegt vornehmlich darin, daß Frauen in marktgängige Fertigkeiten investieren, und nicht etwa Männer sich mehr und mehr im Haushaltssektor spezialisieren - das „eine Mitglied eines effizienten Haushaltes" ist im allgemeinen die Frau.

Eine Teilzeitbeschäftigung könnte nun die mit der Dauer von Erwerbsunterbrechungen kontinuierlich zunehmende Entwertung des Humankapitals verhindern, zumindest aber abschwächen. Mit einer Teilzeitbeschäftigung wird neben der Entwertung des Humankapitals auch die durch Verzicht auf Vollzeiterwerbstätigkeit erlittene Einkommenseinbuße kompensiert. Durch Aufnahme einer Teilzeitarbeit würden infolgedessen die Investitionen sowohl für die Erwerbstätigkeit als auch für den privaten Haushalt optimiert. Das Gesagte gilt um so mehr, wenn nicht lediglich ein 2-Personen-Haushalt zu versorgen ist, sondern zusätzlich Kinder zu betreuen sind. Allgemein wird angenommen, daß der für die Kinderbetreuung erforderliche Aufwand mit dem Alter der Kinder sinkt. Die für den Haushalt aufgebrachte Zeit weist demzufolge ihren höchsten Wert auf, solange Kleinkinder zu betreuen sind. Mit zunehmendem Alter der Kinder, d. h. mit zunehmender Entlastung der Eltern, wird die Teilzeit-Alternative attraktiver.

Neben der Bedeutung der reduzierten Arbeitszeit für die Synchronisation familiärer und beruflicher Aufgaben dürfen zwei weitere Punkte nicht außer acht gelassen werden: die Attraktivität bzw. materielle Ausstattung von Teilzeitarbeitsplätzen und ihre Verbindung zum System sozialer Absicherung. Dies ist um so wichtiger, als gerade die Verdienstmöglichkeiten und die soziale Absicherung von Teilzeitbeschäftigten international beträchtlich variieren. Über den Charakter von Teilzeitstellen hat es eine breite Debatte gegeben, die wir an dieser Stelle nicht auffächern möchten. Festgehalten werden soll jedoch, daß Teilzeitarbeit nicht grundsätzlich mit ,schlechter“ Arbeit gleichgesetzt werden darf. Dies zeigt sich unter anderem daran, daß einige Teilzeitstellen eigens geschaffen werden, um bestimmte Arbeitnehmer, die aufgrund ihrer Lebensumstände das Bedürfnis nach Teilzeitarbeit äußern, im Betrieb zu halten (Tilly 1991). Solche Arrangements kommen allerdings meist im Bereich des Primärarbeitsmarktes vor und stellen wohl eher die Ausnahme von der Regel dar, denn die große Mehrzahl der amerikanischen Teilzeitarbeitsplätze zeichnet sich durch 
niedrige Bezahlung, fehlende Aufstiegsmöglichkeiten und hohe Fluktuation aus. In der Regel sind in den USA die Löhne für Teilzeitstellen niedrig. 1991 verdienten dort Teilzeitbeschäftigte pro Stunde im Durchschnitt gerade 58 Prozent des Stundenlohns eines Vollzeitbeschäftigten (Tilly 1991). Überdies erhalten Teilzeitkräfte in den Vereinigten Staaten weitaus weniger Lohnnebenleistungen und sind nur selten in soziale Sicherungssysteme eingebunden (Kalleberg/Rosenfeld 1990; Harris 1993). Wenn jedoch insbesondere keine Krankenversicherung durch die Berufstätigkeit ermöglicht wird, dürfte dies die Aufnahme von Teilzeitarbeit besonders für die Frauen negativ beeinflussen, die nicht durch ihren Ehemann versichert sind.

Umstritten ist, ob sich hinter der gestiegenen Frauenerwerbstätigkeit primär ein Kohorten- oder aber ein Periodeneffekt verbirgt. Goldin (1990) hat für die USA gezeigt, daß sukzessive Geburtskohorten in allen Altersstufen eine höhere Erwerbsquote aufweisen als ihre Vorgängerkohorten. Ähnliches wurde für Deutschland beobachtet, wobei als zentrale Erklärung die Veränderungen im traditionellen Rollenmodell gegeben wurde. die bei Frauen jüngerer Geburtsjahrgängen einsetzen und auch die späteren Lebenslaufphasen entscheidend prägen (Müller 1983: 71). Demgegenüber kann eine gestiegene Frauenerwerbstätigkeit als periodenbedingte Folge der strukturellen Veränderung des Arbeitsmarktes gedeutet werden, die allen Frauen, unabhängig von ihrem Alter und der Phase im Lebenszyklus, vermehrt Erwerbsmöglichkeiten offeriert hat, wie zum Beispiel durch die Tertiarisierung des Beschäftigungssystems. $\mathrm{Zu}$ denken wäre im weiteren an die gestiegene Nachfrage nach Arbeitskräften unter den günstigen wirtschaftlichen Bedingungen der Nachkriegszeit, was für Deutschland als entscheidend angesehen wurde (Willms-Herget 1985).

Aufgrund der theoretischen Überlegungen erwarten wir, daß Erwerbsunterbrechungen in der Kohortenfolge sowie mit höherer Bildung für weibliche Erwerbsverläufe weniger typisch geworden sind. Weiterhin spricht sowohl die soziologische Perspektive des Rollenkonfliktes als auch die ökonomische Theorie bezüglich weiblicher Erwerbsbiographien für eine Verknüpfung zwischen Familie und Teilzeitarbeit. Wir überprüfen die $\mathrm{Hy}$ pothese, daß sich bei Frauen die Neigung, aufgrund familiärer Verpflichtungen Arbeitsplätze zu verlassen, bei Vollzeitbeschäftigten höher liegt als bei Teilzeitbeschäftigten. Was die Rückkehr in das Beschäftigungssystem nach Erwerbsunterbrechungen anbelangt, so erwarten wir, daß Übergän- ge in Teilzeitarbeit gefördert werden, sobald alle Kinder im Schulalter sind.

\section{Vorteile individueller Längsschnittdaten}

Querschnittsdaten und aggregierte Informationen aus Zeitreihen führen in der Analyse sozialen Wandels häufig zu Artefakten (vgl. Goldin 1990; Murphy 1991; Blossfeld 1995). ${ }^{2}$ So kann es durchaus der Fall sein, daß die Frauenerwerbsquote fällt, obwohl das Ausmaß der weiblichen Erwerbsbeteiligung steigt: beispielsweise wenn die Erwerbsquote aufgrund einer veränderten Altersstruktur sinkt und gleichzeitig jüngere Geburtskohorten wegen einer verlängerten Ausbildungszeit später in das Beschäftigungssystem eintreten (Handl 1988: 39). Bloße Erwerbsquoten sind folglich kaum sinnvoll zu interpretieren, da sie strukturelle Entwicklungen reflektieren, die in unterschiedliche Richtungen wirken und nur mithilfe individueller Längsschnittdaten zu erkennen sind. Im folgenden sollen die substantiellen Interpretationsprobleme anhand der Beziehung von Alter und Arbeitsmarktpartizipation verdeutlicht werden. Frühe Studien über Frauenerwerbstätigkeit postulierten auf Basis der nach Lebensalter M-förmig verlaufenden Frauenerwerbsquote typische Phasen der weiblichen Erwerbsbeteiligung. Zwischen Beendigung der Ausbildung und Heirat bzw. Geburt des ersten Kindes wurde kontinuierliche Erwerbstätigkeit als Regelfall identifiziert. Für Altersstufen nach der Familiengründung wurde eine Unterbrechung der Erwerbstätigkeit für die Dauer von fünf bis zehn Jahren beobachtet. Diesem Zeitabschnitt folgte nach Einschulung des jüngsten Kindes oft die Wiederaufnahme der Erwerbstätigkeit - zunächst eher diskontinuierlich, und wenn alle Kinder erwachsen waren, oftmals wieder in permanenter Form (Stromberg/Harkess 1978).

Betrachtet man für Zehnjahresintervalle seit 1940 die US-Frauenerwerbsquote als Funktion des

Die Unangemessenheit aggregierter Querschnittsdaten wurde in überzeugender Weise von Jacobs (1989) gezeigt: Während auf der Makroebene eine im Zeitverlauf überraschend stabile geschlechtsspezifische Segregation des USamerikanischen Arbeitsmarktes zu beobachten ist. findet im tatsächlichen Arbeitsmarktverhalten von Frauen ein großes Ausmaß an Wechseln zwischen von Männern dominierten, relativ geschlechtsneutralen und typisch weiblichen Berufen statt. Dieses scheinbare Paradox kann nur verstanden werden, wenn Erwerbsverhalten auf der individuellen Ebene in einer Längsschnittperspektive untersucht wird. 


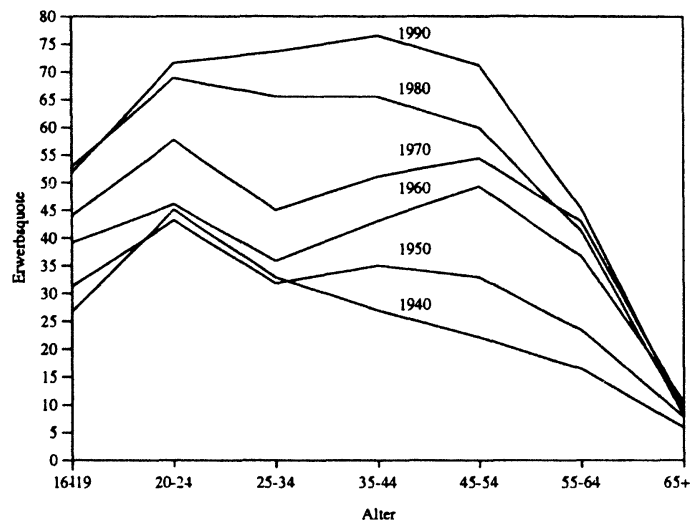

Abb. 2 Anteil der Erwerbspersonen (civilian labor force) bei US-amerikanischen Frauen für verschiedene Jahre.

Frauen ab 16 Jahre (bzw. ab 14 Jahre bis 1960) in Privathaushalten.

Quelle: Statistical Abstract of the United States, diverse Ausgaben (basierend auf Current Population Survey).

Merkmals Alter (Abbildung 2), so zeigt sich der erwähnte $M$-förmige Kurvenverlauf lediglich für ungefähr zwanzig Jahre, von Mitte der 50er bis Mitte der 70er Jahre. Zunächst steigt die Erwerbsbeteiligung bis zum Alter von 20 bis 24 Jahren an, dann geht sie zurück und erreicht einen Tiefpunkt im Alter zwischen 25 und 34 Jahren. Danach steigt die Partizipationsrate erneut, bis sie ab einem Alter von 45 bis 54 Jahren wieder abfällt. Bereits für 1980 ergibt sich jedoch eine beträchtliche Veränderung im Kurvenverlauf. Zwar steigt die Erwerbsquote nach wie vor bis zum Alter von 20 bis 24 Jahren, danach bleibt jedoch der auf familiäre Bedingungen zurückgeführte Einschnitt aus (vgl. auch Waite 1981), und im Jahre 1990 ist das Muster vollständig verschwunden. Anhand dieser Abbildung wird deutlich, daß die oft als typisch bezeichnete M-Kurve weiblichen Erwerbsverhaltens offenbar nur für eine relativ kurze Zeit charakteristisch war.

Schlüsse aus diesen Veränderungen auf die ihnen zugrundeliegenden Mechanismen (wie beispielsweise die Familienbedingtheit weiblichen Erwerbsverhaltens) sind kaum möglich, da ohne das Vorliegen individueller Längsschnittdaten überaus verschiedene Erklärungen möglich sind. Unter Umständen spiegeln sich in den Altersmustern Veränderungen in der Ausbildungszeit oder dem Qualifikationsniveau der Frauen wider, Veränderungen des Heiratszeitpunktes oder abnehmende
Geburtenraten, die eine frühere Rückkehr in das Erwerbssystem ermöglichen, oder ein verändertes Erwerbsverhalten jüngerer Geburtsjahrgänge generell. Teachman et al. (1987) vermuten, daß die nach dem 2. Weltkrieg geborenen Frauen ein neues Erwerbstätigkeitsprofil im Lebenszyklus zeigen. Wie ein Blick in die Arbeitsmarktstatistik belegt, ist die Frauenerwerbstätigkeit überproportional bei verheirateten Frauen mit Kindern gestiegen, insbesondere bei Frauen mit Vorschulkindern (Tabelle 3).

Tabelle 3: Erwerbsquote (civilian labor force) bei verheirateten und mit dem Ehemann zusammenlebenden Frauen, nach Alter der Kinder.

\begin{tabular}{llll}
\hline Jahr & $\begin{array}{l}\text { Kein Kind } \\
\text { unter 18 }\end{array}$ & $\begin{array}{l}\text { Nur Kind(er) } \\
\text { von } 6 \text { bis 17 }\end{array}$ & $\begin{array}{l}\text { Kind(er) } \\
\text { unter 6 }\end{array}$ \\
\hline 1950 & 30,3 & 28,3 & 11,9 \\
1960 & 34,7 & 39,0 & 18,6 \\
1970 & 42,2 & 49,2 & 30,3 \\
1980 & 46,0 & 61,7 & 45,1 \\
1990 & 51,1 & 73,6 & 58,9 \\
1992 & 51,9 & 75,4 & 59,9 \\
\hline
\end{tabular}

Frauen ab 16 Jahre (bzw. ab 14 Jahre bis 1960) in Privathaushalten.

Quelle: Statistical Abstract of the United States, diverse Ausgaben (basierend auf Current Population Survey).

Aber auch diese Prozentwerte können auf völlig unterschiedliche Art und Weise zustandegekommen sein und nur mithilfe individueller Längsschnittdaten entschlüsselt werden. Die Partizipationsrate von 59,9 Prozent der verheirateten Frauen mit Kindern unter sechs Jahren für das Jahr 1992 kann zum Beispiel bedeuten, daß 59,9 Prozent dieser Frauen kontinuierlich erwerbstätig und die restlichen 40,1 Prozent immer Hausfrauen waren. Möglich ist aber auch, daß alle Frauen zwischen Erwerbstätigkeit und Nichterwerbstätigkeit hin- und herwechselten, also 100 Prozent irgendwann einmal in diesem Jahr erwerbstätig waren. Nimmt man die Information hinzu, daß im gleichen Jahr 51,9 Prozent der verheirateten Frauen ohne Kinder unter 18 Jahren erwerbstätig waren. so sagt dies immer noch nichts über den tatsächlichen Effekt aus, den die Gegenwart von Kindern auf Erwerbstätigkeit ausübt. Möglicherweise ist die Partizipationsrate der zuletzt genannten Frauen nur deshalb niedriger, weil diese Frauen im 
Durchschnitt älter sind und im Alter die Erwerbstätigkeit abnimmt. Möglicherweise ist auch ein Kohorteneffekt verantwortlich, das heißt, es handelt sich um Geburtsjahrgänge mit einer niedrigeren Erwerbsbeteiligung während ihres gesamten Arbeitslebens.

Zusammenfassend kann festgehalten werden, daß die Aussagekraft im Hinblick auf sozialen Wandel äußerst begrenzt ist, sofern die offenkundige Zeitabhängigkeit sozialer Prozesse ignoriert wird und keine konzeptionelle Trennung zwischen Alters-, Perioden- und Kohorteneffekten erfolgt. Erst von einer Längsschnittanalyse mit Individualdaten, die dieses ermöglicht, können Einblicke in Mechanismen sozialen Wandels erwartet werden, vorausgesetzt, es liegen Informationen über die Angehörigen verschiedener Geburtskohorten für einen längeren Zeitraum vor.

\section{Datenbasis und Methoden}

Die folgenden Längsschnittanalysen basieren auf Daten des „National Survey of Families and Households (NSFH)“. Retrospektiv erhobene Informationen zur Familiengeschichte, zu Veränderungen in Haushaltsstrukturen und Erwerbsbiographien machen den Datensatz für die vorliegende Fragestellung besonders geeignet. In der Erhebung wurden 1987/1988 auf Grundlage einer $\mathrm{Zu}$ fallsauswahl über 13000 Personen befragt. Ein Mitglied pro Haushalt wurde per Zufall für eine mündliche Befragung ausgewählt (primary respondents), ggf. wurden ein zweites und drittes Haushaltsmitglied um Teilnahme an einer kürzeren Befragung gebeten. In unsere Analysen wurden nur primary respondents einbezogen, und zwar weiße Frauen ab 19 Jahren. ${ }^{3}$

\footnotetext{
Besonders in den fünfziger und sechziger Jahren lag die Erwerbsbeteiligung schwarzer Frauen signifikant höher als die weißer Frauen. Seither hat sich der Unterschied mehr und mehr verringert, und 1991 lag die Erwerbsquote weißer Frauen zum ersten Mal höher (Economic Report of the President 1992). Nach Arbeitsmarktprognosen für die nächsten zehn Jahre wird die Erwerbsbeteiligung weiBer Frauen auf einem höheren Niveau bleiben. Die unterschiedliche Entwicklung verbietet eine gemeinsame Analyse ebenso wie die abweichenden Muster der Erwerbsbeteiligung im Lebenszyklus. Beispielsweise hatten bis Ende der achtziger Jahre weiße Frauen im Alter von 16 bis 19 Jahren eine höhere Partizipationsrate als ältere Frauen, bei schwarzen Frauen war es umgekehrt. 1991 betrug die Erwerbsrate weißer Frauen im Alter von 16-19 Jahren $54.3 \%$ und bei den über 20 jährigen $57.7 \%$. Die schwarzen Frauen wiesen dagegen in der Altersgruppe 16-19 eine
}

Eine Erwerbsbiographie beginnt im vorliegenden Datensatz mit dem Zeitpunkt, zu dem die betreffende Person das erste Mal mindestens sechs Monate lang ununterbrochen erwerbstätig war. Der Erwerbsverlauf ist als Folge von Episoden dargestellt, die durch die Ereignisse Beendigung bzw. Wiederaufnahme einer Erwerbstätigkeit definiert sind. Die Dauer einer jeden aus dieser Definition resultierenden Episode wurde in Monaten codiert, und für jede der Erwerbstätigkeitsepisoden ist bekannt, ob die Befragte während dieser Zeit hauptsächlich vollzeit- oder teilzeitbeschäftigt war. ${ }^{4}$ Aufgrund der Vorgaben in den Daten ist es weder möglich, direkte Übergänge zwischen Vollzeitund Teilzeiterwerbstätigkeit zu operationalisieren, ${ }^{5}$ noch in den Nichterwerbstätigkeits-Episoden eine Differenzierung zwischen den Zuständen "Hausfrau“ und „arbeitslos" vorzunehmen. Daher beschränkt sich die Analyse auf Übergänge von einer Phase hauptsächlicher Vollzeiterwerbstätigkeit zu Nichterwerbstätigkeit (VZ $>N E$ ), analog von Teilzeit-zu Nichterwerbstätigkeit (TZ $>N E$ ). von Nichterwerbs- $z u$ Vollzeiterwerbstätigkeit $(\mathrm{NE}>\mathrm{VZ}$ ) und von Nichterwerbs- zu Teilzeiterwerbstätigkeit (NE > TZ). Die Rekonstruktion lückenloser Erwerbsbiographien von Beginn der regulären Erwerbstätigkeit bis hin zum Befragungszeitpunkt war für 4120 weiße Frauen möglich. ${ }^{6}$

Erwerbsquote von lediglich $33,5 \%$ auf, in der Altersgruppe über 20 jedoch $59,3 \%$ (Economic Report of the President 1992; vgl. auch Mellor/Parks 1988).

4 Abweichend zur Definition des U.S. Burcau of Labor Statistics, das von einer Vollzeittätigkeit von 35 oder mehr Wochenarbeitsstunden ausgeht, ist im NSFH-Datensatz die Grenze bei 30 Stunden gezogen worden und Teilzeitarbeit wird definiert als eine Tätigkeit mit weniger als 30 Wochenstunden. Die durchschnittliche Dauer von Teilzeitepisoden beträgt 4,5 Jahre gegenüber mehr als 7 Jahren bei Vollzeitepisoden. Mehr als $30 \%$ der Teilzeitepisoden dauern nur 12 Monate oder kürzer, die Hälfte dieser Episoden ist bereits nach 26 Monaten beendet. Nur $16 \%$ der Vollzeitepisoden aber dauern ein Jahr oder weniger und ihr Median beträgt 53 Monate.

'Diese Begrenzung ist jedoch nicht entscheidend, da nach bisherigen Untersuchungen für Vollzeit- wie auch Teilzeiterwerbstätige Übergänge in die Nichterwerbstätigkeit weitaus wahrscheinlicher sind als Wechsel zwischen den beiden genannten Zuständen (vgl. Blank 1989; Moen/Smith 1986: 469).

${ }^{6}$ Aus dem Sample wurden alle Frauen ausgeschlossen, die nie erwerbstätig gewesen sind bzw. nie eine Erwerbstätigkeit von wenigstens 6 Monaten ausgeübt haben. Episoden, die ab dem Alter von 60 Jahren beginnen, wurden von den Berechnungen ausgeschlossen, um Effekte der Erwerbstätigkeit im Rentenalter zu minimieren; und es 
Zur Analyse der Übergangsraten zwischen den genannten Zuständen wurde das Instrumentarium der Ereignisanalyse herangezogen (Blossfeld et al. 1989; Tuma/Hannan 1984; Allison 1984; Kalbfleisch/Prentice 1980). Abhängige Variable ist hierbei die Übergangsrate von Zustand $\mathrm{j}$ zu Zustand $\mathrm{k}$ :

$r_{j k}(t)=\lim _{\Delta t} \cdot \frac{1}{\Delta t} \quad P_{j k}(t \leq T<t+\Delta t \quad l t \leq T), j \neq k$

$r_{1 k}$ bezeichnet die „Neigung“, den Zustand j zugunsten des Zustandes k zu verlassen, vorausgesetzt, eine Person befand sich zum Zeitpunkt $t$ noch im Zustand j, war also gleichsam , at risk“. Eine solche Übergangsrate kann als bedingte Wahrscheinlichkeit für das Eintreten des Zustandswechsels im Zeitintervall $[t, t+\Delta t]$ interpretiert werden. Die Modellparameter wurden durch die Maximum Likelihood-Methode geschätzt, die es erlaubt, zensierte Episoden in die Analyse miteinzubeziehen. Zur Schätzung der Übergangsraten verwenden wir ein Exponentialmodell sowie - um Effekte der Zustandsdauer zu kontrollieren - ein Piecewise-constant-Modell. Mit einem Piecewise-constant-Modell werden für definierte Zeitabschnitte der Episodendauer spezifische Übergangsraten geschätzt (die innerhalb der Zeitabschnitte jedoch konstant bleiben), wobei der Vorteil darin liegt, daß es sich um ein nicht-parametrisches Modell handelt, die Ratenfunktion also nicht spezifiziert werden muß. Statt einer einzigen Konstante wie im regulären Exponentialmodell erhält man spezifische Konstanten für die im einzelnen definierten Verweildauerabschnitte.

Während in Querschnittsanalysen interdependente Ereignisse nicht auf Ursache und Wirkung hin betrachtet werden können, weil Angaben über das zeitliche Eintreten fehlen, ${ }^{7}$ wird in vorliegender Längsschnittanalyse die tatsächliche zeitliche $\mathrm{Ab}$ folge der Ereignisse modelliert. Die vorgenommene Operationalisierung familiärer Ereignisse unterscheidet sich insofern vom klassischen Familienzyklus-Modell (Glick 1977), als daß nicht ein normatives Familienbild unterstellt werden muß. Wenn beispielsweise die Geburt eines Kindes der Heirat vorangeht, wird dem modelltechnisch ebenso Rechnung getragen wie wenn Personen mehrmals geheiratet haben. Die Einflüsse einer

wurden nur Episoden einbezogen, die nach 1949 beginnen. Ausfälle ergaben sich dadurch, daß für $8,7 \%(n=395)$ dieser Zielpopulation $(n=4515)$ die Erwerbs- oder Familienbiographie nicht vollständig vorliegt.

7 Zur Kausalitätsproblematik vgl. die Debatte bei Cramer (1980) und Bernhardt (1993) über die kausale Beziehung zwischen Fertilität und Frauenerwerbstätigkeit.
Ehe wurden durch zwei Dummyvariablen erfaßt:

- verheiratet ohne Kind,

- verheiratet mit Kind(ern),

- nicht verheiratet als Referenzkategorie.

Für die Auswirkungen der Kinderbetreuung wurden vier Konstellationen unterschieden:

- Vorschulkind (mindestens ein Kind jünger als 6 Jahre im Haushalt),

- Schulkind (kein Vorschulkind, jedoch mindestens ein Kind von 6 bis 18 Jahre im Haushalt),

- Kind(er) mindestens 18 als Proxyvariable für die Situation, daß alle Kinder den betreffenden Haushalt verlassen haben,

- keine Kinder als Referenzkategorie dieser drei Dummyvariablen.

Solange eine Ehe existiert, die Frau bisher jedoch kein Kind geboren hat, wird die Variable ,verheiratet ohne Kind" mit "1" codiert. Mit der Geburt des ersten Kindes wird dieser Variable die Ausprägung „0“ zugewiesen und die Variable „verheiratet mit Kind(ern)" nimmt den Wert „1" an (die gleiche Codierung wird vorgenommen, wenn bereits vor einer Ehe mindestens ein Kind vorhanden ist). Der Zweck dieser Unterscheidung liegt darin, die Effekte von Ehe ,an sich", von Kindern bei verheirateten Müttern und von Kindern bei unverheirateten Müttern zu separieren. ${ }^{8}$ So ergibt sich der Vorschulkind-Effekt für unverheiratete Mütter aus dem Vergleich des Koeffizienten für ,Vorschulkind" mit der Baseline, für verheiratete Mütter muß man den Effekt ,verheiratet mit Kin$\mathrm{d}(\mathrm{ern})$ " hinzuaddieren.

Bildung wurde in Schuljahren gemessen und als zeitkonstantes Merkmal behandelt. Geburtskohorten und Zeitperioden werden durch Dummyvariablen repräsentiert, die jeweils einander benachbarte Jahre umfassen. Die Einteilung der Perioden erfolgte gemäß ihrer sequentiellen Abfolge. Für spezielle Abgrenzungen sahen wir keine Notwendigkeit, da es keine abrupten Veränderungen in der Nachkriegsentwicklung des amerikanischen Arbeitsmarktes gab. Um die Effekte der zeitabhängigen Kovariablen auf die Übergangsraten zu schätzen, wurden die Episoden so gesplittet.

\footnotetext{
${ }^{8} 1960$ lebten in den USA $9 \%$ aller Kinder unter 18 Jahre mit nur einem Elternteil, 1990 waren es bereits $24 \%$ aller Kinder. Es wird geschätzt, daß von den 1980 geborenen Kindern 70\% (weiße) bzw. $94 \%$ (schwarze) bis zur Vollendung des 17. Lebensjahrs wenigstens zeitweise in einem Haushalt mit nur einem Elternteil wohnen (Popenoc 1993: 531).
} 
Vollzeit > Nichterwerbstatigkeit

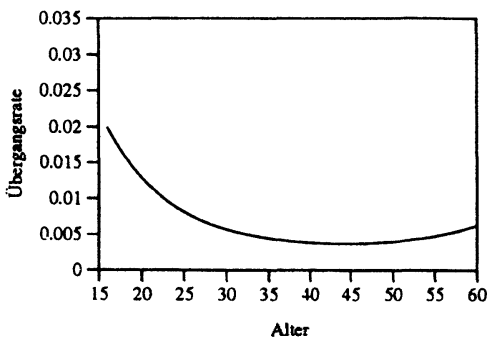

Nichterwerbstatigkeit > Vollzeit

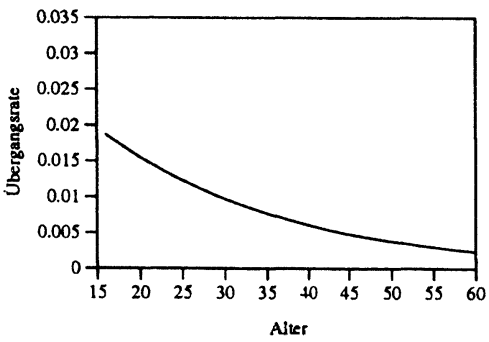

Alter
Teilzeit > Nichterwertstatigkeit

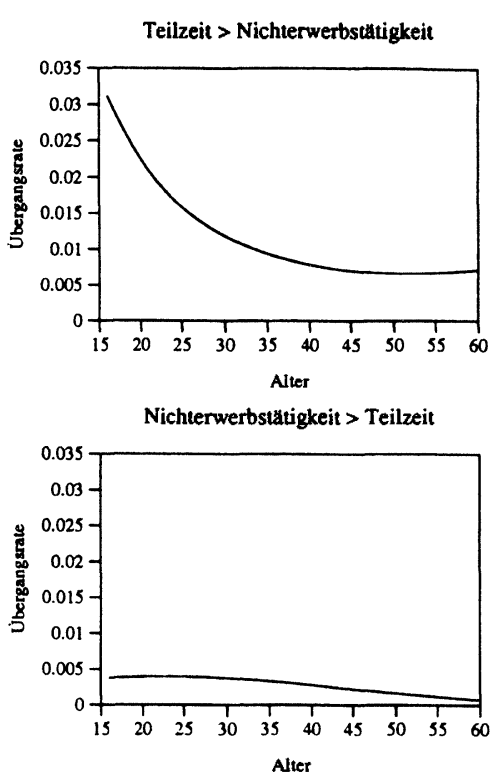

Nichterwerbstătigkeit > Teilzeit

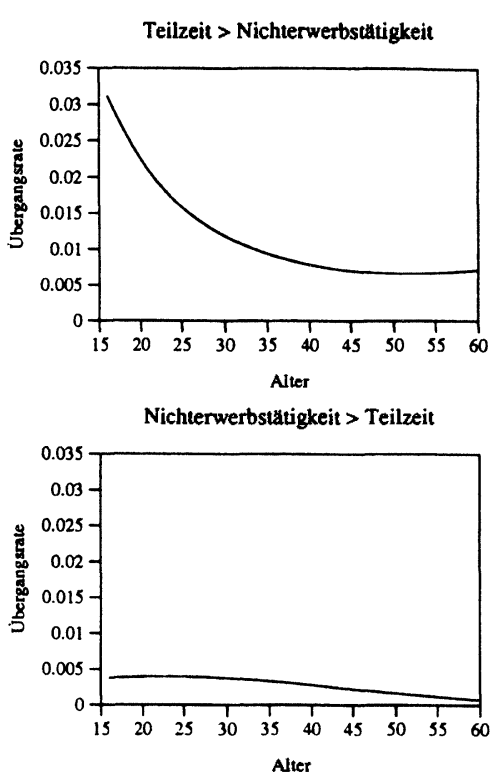

Abb. 3 Übergangsraten in Abhängigkeit vom Lebensalter.

Eigene Berechnungen, NSFH-Daten

daß sich Subepisoden mit einer Dauer von höchstens einem Jahr ergaben. Dieses Episodensplitting führte bei 7448 Ereignissen $\mathrm{zu}$ insgesamt 93960 Subepisoden als Einheiten für die Analyse der Übergangsraten.

\section{Ergebnisse ${ }^{9}$}

\subsection{Alter, Kohorte, Periode}

In Abbildung 3 sind die altersspezifischen Übergangsraten dargestellt, wie sie aus einem Exponentialmodell berechnet wurden, welches lediglich die Effekte für „Alter“ und „Alter quadriert" schätzt. Die Übergangsrate von Teilzeitbeschäftigung zu Nichterwerbstätigkeit ist über die gesamte Spannweite des Alters hinweg höher als jene von Vollzeitbeschäftigung zu Nichterwerbstätigkeit. Beide Kurven haben einen nicht-linearen Verlauf: Erwerbsunterbrechungen kommen in relativ jungen Jahren überdurchschnittlich häufig vor, wobei es mit zunehmendem Lebensalter mehr und mehr unwahrscheinlich wird, den Arbeitsmarkt zu verlassen, und ab einem gewissen Alter steigt die Rate leicht an. Das Minimum der altersabhängi-

\footnotetext{
"Alle Modelle wurden mit dem neuentwickelten Programmpaket TDA (Transition Data Analysis) von Götz. Rohwer (Universität Bremen) geschätzt. das elaborierte Längsschnittanalysen ermöglicht (Rohwer 1994). Wir danken Götz. Rohwer darüber hinaus für seine Hilfe bei der Datenaufbereitung.
}

gen Übergangsrate errechnet sich mit 44 (Vollzeitbeschäftigung) bzw. 52 (Teilzeitbeschäftigung) Jahren. In Deutschland zeigt sich im Übergang von Vollzeitarbeit zu Nichterwerbstätigkeit ein völlig anderes Bild. Die Rate liegt von Beginn an durchgängig höher als in den USA, außerdem steigt sie in Deutschland noch bis zum Alter von etwa 24 Jahren und fällt erst danach ab (Blossfeld/ Rohwer 1995).

Im Übergang von Nichterwerbstätigkeit in Beschäftigungsverhältnisse nimmt die Rate von Nichterwerbstätigkeit nach Vollzeiterwerbstätigkeit mit dem Lebensalter monoton ab. Nichterwerbstätige Frauen gelangen mit zunehmendem Alter immer scltener in Vollzeitstellen. Beispielsweise liegt die Wahrscheinlichkeit einer 20jährigen Frau viermal höher als die einer 50jährigen. Die entsprechende Rate von Nichterwerbstätigkeit zu Teilzeitbeschäftigung ist demgegenüber während des gesamten Lebensalters extrem niedrig. Im Gegensatz zu den drei anderen Kurven steigt diese Rate - allerdings kaum merklich - bis zum Alter von 23 Jahren an, was auf eine etwas größere Bedeutung von Teilzeitarbeit zu Beginn der Berufslaufbahn schließen läßt. Hinsichtlich einer Wiederaufnahme der Erwerbstätigkeit unterscheidet sich in Deutschland vor allem die Übergangsrate nach Teilzeitarbeit. Diese Rate liegt viel höher als in den USA und steigt bis zu ihrem Maximum bei ca. 32 Jahren merklich an (Blossfeld/ Rohwer 1995). 
Tabelle 4: Die Effekte von Alter, Periode und Kohorte auf die geschätzten Übergangsraten (Standardfehler in Klammern). Exponentialmodell.

\begin{tabular}{|c|c|c|c|c|}
\hline & $V Z>N E$ & $T Z>N E$ & $N E>V Z$ & $N E>T Z$ \\
\hline Konstante & $\begin{array}{c}-0.9031^{\star \star} \\
(0.2876)\end{array}$ & $\begin{array}{c}-1.5997^{\star *} \\
(0.6009)\end{array}$ & $\begin{array}{c}-4.1520^{\star \star} \\
(0.4528)\end{array}$ & $\begin{array}{c}-9.0629^{\star \star} \\
(0.8006)\end{array}$ \\
\hline Alter & $\begin{array}{c}-0.1957^{\star \star} \\
(0.0108)\end{array}$ & $\begin{array}{c}-0.1129^{\star \star} \\
(0.0217)\end{array}$ & $\begin{array}{c}0.0248 \\
(0.0183)\end{array}$ & $\begin{array}{c}0.1010^{\star \star} \\
(0.0299)\end{array}$ \\
\hline Alter quadriert & $\begin{array}{c}0.0022^{* *} \\
(0.0001)\end{array}$ & $\begin{array}{l}0.0010^{* *} \\
(0.0002)\end{array}$ & $\begin{array}{c}-0.0012^{\star *} \\
(0.0002)\end{array}$ & $\begin{array}{c}-0.0012^{\star \star} \\
(0.0003)\end{array}$ \\
\hline Kohorte 1924-1928 & $\begin{array}{c}-0.2353^{\star} \\
(0.1099)\end{array}$ & $\begin{array}{c}-0.0445 \\
(0.2289)\end{array}$ & $\begin{array}{c}-0.5909^{\star *} \\
(0.1690)\end{array}$ & $\begin{array}{c}0.4376 \\
(0.2958)\end{array}$ \\
\hline Kohorte 1929-1933 & $\begin{array}{c}-0.1957 \\
(0.1280)\end{array}$ & $\begin{array}{c}-0.3185 \\
(0.2918)\end{array}$ & $\begin{array}{c}-0.6314^{\star \star} \\
(0.1838)\end{array}$ & $\begin{array}{c}0.3829 \\
(0.3413)\end{array}$ \\
\hline Kohorte 1934-1938 & $\begin{array}{c}-0.2095 \\
(0.1452)\end{array}$ & $\begin{array}{c}-0.2451 \\
(0.3130)\end{array}$ & $\begin{array}{c}-0.7694^{\star \star} \\
(0.2130)\end{array}$ & $\begin{array}{c}0.7540^{\star} \\
(0.3818)\end{array}$ \\
\hline Kohorte 1939-1943 & $\begin{array}{c}-0.2866 \\
(0.1721)\end{array}$ & $\begin{array}{c}-0.0577 \\
(0.3670)\end{array}$ & $\begin{array}{c}-0.6924^{\star \star} \\
(0.2556)\end{array}$ & $\begin{array}{c}1.0615^{\star} \\
(0.4495)\end{array}$ \\
\hline Kohorte 1944-1948 & $\begin{array}{c}-0.1449 \\
(0.1987)\end{array}$ & $\begin{array}{c}-0.1887 \\
(0.4274)\end{array}$ & $\begin{array}{r}-0.7101^{\star} \\
(0.2987)\end{array}$ & $\begin{array}{l}1.3493^{\star *} \\
(0.5183)\end{array}$ \\
\hline Kohorte 1949-1953 & $\begin{array}{c}-0.2559 \\
(0.2259)\end{array}$ & $\begin{array}{c}-0.0047 \\
(0.4783)\end{array}$ & $\begin{array}{c}-0.6623 \\
(0.3397)\end{array}$ & $\begin{array}{l}1.5759^{\star *} \\
(0.5844)\end{array}$ \\
\hline Kohorte 1954-1958 & $\begin{array}{c}-0.3783 \\
(0.2565)\end{array}$ & $\begin{array}{c}0.0326 \\
(0.5437)\end{array}$ & $\begin{array}{r}-0.7888^{*} \\
(0.3848)\end{array}$ & $\begin{array}{c}1.9590^{\star \star} \\
(0.6555)\end{array}$ \\
\hline Kohorte 1959-1963 & $\begin{array}{c}-0.5050 \\
(0.2879)\end{array}$ & $\begin{array}{c}-0.0965 \\
(0.6089)\end{array}$ & $\begin{array}{c}-0.7042 \\
(0.4319)\end{array}$ & $\begin{array}{l}1.9974^{\star \star} \\
(0.7313)\end{array}$ \\
\hline Kohorte 1964-1969 & $\begin{array}{c}-0.3367 \\
(0.3217)\end{array}$ & $\begin{array}{c}0.0666 \\
(0.6608)\end{array}$ & $\begin{array}{c}-0.5957 \\
(0.4812)\end{array}$ & $\begin{array}{l}2.2763^{\star \star} \\
(0.8081)\end{array}$ \\
\hline Periode 1958-1962 & $\begin{array}{c}-0.1305 \\
(0.1015)\end{array}$ & $\begin{array}{c}-0.1225 \\
(0.2274)\end{array}$ & $\begin{array}{c}-0.0348 \\
(0.1636)\end{array}$ & $\begin{array}{c}0.1798 \\
(0.3973)\end{array}$ \\
\hline Periode 1963-1967 & $\begin{array}{c}-0.2070 \\
(0.1143)\end{array}$ & $\begin{array}{c}-0.5146^{\star} \\
(0.2498)\end{array}$ & $\begin{array}{c}0.2781 \\
(0.1770)\end{array}$ & $\begin{array}{c}0.3677 \\
(0.3998)\end{array}$ \\
\hline Periode 1968-1972 & $\begin{array}{c}-0.1216 \\
(0.1305)\end{array}$ & $\begin{array}{c}-0.2303 \\
(0.2746)\end{array}$ & $\begin{array}{c}0.4728^{*} \\
(0.2064)\end{array}$ & $\begin{array}{c}0.1319 \\
(0.4358)\end{array}$ \\
\hline Periode 1973-1977 & $\begin{array}{c}-0.1528 \\
(0.1535)\end{array}$ & $\begin{array}{c}-0.3638 \\
(0.3224)\end{array}$ & $\begin{array}{c}0.6584^{\star \star} \\
(0.2409)\end{array}$ & $\begin{array}{c}0.1372 \\
(0.4792)\end{array}$ \\
\hline Periode 1978-1982 & $\begin{array}{c}-0.0432 \\
(0.1787)\end{array}$ & $\begin{array}{c}-0.3933 \\
(0.3726)\end{array}$ & $\begin{array}{l}0.7977^{\star \star} \\
(0.2812)\end{array}$ & $\begin{array}{c}0.1702 \\
(0.5335)\end{array}$ \\
\hline Periode 1983-1988 & $\begin{array}{c}0.1127 \\
(0.2059)\end{array}$ & $\begin{array}{c}-0.2956 \\
(0.4260)\end{array}$ & $\begin{array}{l}0.8309^{\star *} \\
(0.3218)\end{array}$ & $\begin{array}{c}0.5219 \\
(0.5900)\end{array}$ \\
\hline Ereignisse & 3570 & 904 & 2167 & 807 \\
\hline \multicolumn{5}{|c|}{ Log-likelihood für die simultan geschätzten Übergänge: $-42963,8$ mit $d . f .=18$} \\
\hline
\end{tabular}

** signifikant mit $\mathrm{p}<0,01$

* signifikant mit $p<0,05$

Referenzkategorie Geburtskohorten: < 1924 / Referenzkategorie Zeitperioden: < 1958 
Im nächsten Schritt werden die Effekte von Lebensalter, Geburtskohorte und Zeitperiode simultan betrachtet (zur Problematik der Differenzierung dieser Konzepte siehe Ryder 1985; Hobcraft et al. 1982; Burt 1991; Riley 1987). Die Übergänge in die Nichterwerbstätigkeit erweisen sich noch immer als altersbedingt, gleichgültig, ob vorher vollzeit oder teilzeit gearbeitet wurde (Tabelle 4). Weder die $\mathrm{Zu}$ gehörigkeit zu bestimmten Geburtskohorten, noch die historischen Zeitpunkte, zu denen diese Übergänge stattfanden, vermögen die Unterbrechungsmuster wesentlich zu erklären. Frauen der jüngeren Kohorten unterbrechen ihre Erwerbstätigkeit nicht signifikant seltener, als es bereits bei den älteren Jahrgängen der Fall war. Der aus Querschnittsdaten häufig gezogene Schluß, das Muster der Frauenerwerbstätigkeit würde bald dem der Männer entsprechen (Masnick/Bane 1980), scheint nach diesem Längsschnittergebnis nicht haltbar. Wir müssen stattdessen annehmen, daß sich die seit etwa $20 \mathrm{Jah}$ ren verändernde Erwerbsquote nicht durch einen Verzicht auf Erwerbsunterbrechungen, sondern aus der Tatsache ergeben hat, daß die Frauen der jüngeren Jahrgänge die Erwerbstätigkeit eher wiederaufnehmen.

In der Tat wird diese Annahme gestützt, wenn wir einen Blick auf die Übergänge von Nichterwerbstätigkeit in das Beschäftigungssystem werfen. Die Mitglieder der jüngeren Kohorten neigen signifikant häufiger zur Wiederaufnahme beruflicher Teilzeittätigkeiten als die älteren Geburtsjahrgänge, wie der überaus robuste Trend in der Kohortenfolge zeigt, der sich zudem beschleunigt hat. Frauen der Jahrgänge 1934-1938 gehen mit doppelt so hoher Wahrscheinlichkeit in Teilzeittätigkeiten wie die Angehörigen der Referenzkohorte $(\exp (0.7540)=2,1)$. Bei Frauen, die in den fünfziger Jahren geboren sind, ist diese Wahrscheinlichkeit bereits siebenmal so hoch und bei den Frauen der Geburtsjahrgänge 1964-1969 schließlich fast zehnmal. Die Wechsel von Nichterwerbstätigkeit in Vollzeitbeschäftigung zeigen trotz einiger statistisch signifikanter Koeffizienten keinen systematischen Trend in der Kohortenfolge, ${ }^{10}$ dieser Übergang erweist sich deutlich als ein Periodenphänomen. Seit Anfang der 70er Jahre sind die entsprechenden Übergangsraten unabhängig von Alter und Kohorte gegenüber früheren Jahren beachtlich angestiegen.

\footnotetext{
117 Wenn die Familienvariablen in die Modelle einbezogen werden, sind die Koeffizienten für Kohorten beim Ubergang in Vollzeiterwerbstätigkeit nicht mehr signifikant.
}

\subsection{Bildung}

Wer nicht in Erwerbsarbeit steht, verliert nach und nach seine marktgängigen Fertigkeiten - als Folge sinken mit der Dauer von Erwerbsunterbrechungen die Aussichten auf einen der Qualifikation entsprechenden Arbeitsplatz, und Hausarbeit bietet sich zunehmend als opportune Alternative an. Umgekehrt nimmt mit der im Beschäftigungssystem verbrachten Zeit die Menge der für die weitere Karriere förderlichen Erfahrungen und Ressourcen zu - Beschäftigungsstabilität erhöht auf diese Weise stetig die Kosten dafür, den Arbeitsplatz aufzugeben. Um die Effekte zu kontrollieren, die von der Verweildauer in den betrachteten Zuständen ausgehen, wurde das Piecewise-constant-Modell angewendet (Tabelle 5). Wie erwartet, sinken die Übergangsraten mit der Verweildauer.

Tabelle 5 weist im weiteren die direkten Effekte von Bildung, Heirat und Kindern aus, wie sie sich unter Kontrolle der zeitbezogenen Merkmale (Verweildauer, Alter, Kohorte, Periode) ergeben. Da das Bildungsniveau in der Kohortenfolge anstieg und eine erhöhte Bildung den Arbeitsmark teintritt in höhere Lebensalter verlagert - also die Partizipationsquote der jüngeren Frauen reduziert -, ist es besonders wichtig, die Netto-Effekte von Kohorte und Bildung zu trennen. Die Analyse zeigt deutlich, daß höhere Bildung die Rate für das Verlassen von Vollzeitstellen signifikant reduziert, und zwar für jedes Ausbildungsjahr um 4 Prozent. Die Humankapitaltheorie deutet diesen Sachverhalt in der Weise, daß höher qualifizierte Frauen mit größerer Wahrscheinlichkeit besser bezahlte Tätigkeiten ausüben, was das Verlassen dieser Stellen kostenträchtiger werden läßt. Interessanterweise übt Bildung jedoch keinen signifikanten Effekt auf das Verlassen von Teilzeittätigkeiten aus, was wiederum zeigt, daß es sich bei Vollzeit- und Teilzeitarbeit um grundlegend verschiedene Erwerbstypen handelt. Wenn umgekehrt die Übergänge von Nichterwerbstätigkeit in das Beschäftigungssystem betrachtet werden, so hat hier Bildung positive Effekte in beiden Fällen, der Effekt auf Übergänge in Teilzeitbeschäftigung ist noch etwas stärker als der auf Vollzeitbeschäftigung.

\subsection{Familienzyklus}

Eine Heirat wie auch Kinder gleich welchen Alters erhöhen bei vollzeiterwerbstätigen Frauen signifikant die Wahrscheinlichkeit, den Arbeitsmarkt zu 
Tabelle 5: Die Effekte von Zustandsdauer, Bildung und Familienereignissen auf die geschätzten Übergangsraten unter Kontrolle von Alter, Periode und Kohorte (Standardfehler in Klammern). Piecewise-constant-Modell

\begin{tabular}{|c|c|c|c|c|}
\hline & $V Z>N E$ & $\mathrm{TZ}>\mathrm{NE}$ & $N E>V Z$ & $N E>T Z$ \\
\hline Dauer bis 12 Monate & $\begin{array}{c}-1.0327^{\star \star} \\
(0.3079)\end{array}$ & $\begin{array}{r}-1.5027^{*} \\
(0.6431)\end{array}$ & $\begin{array}{c}-4.9672^{\star \star} \\
(0.4790)\end{array}$ & $\begin{array}{c}-9.7267^{\star *} \\
(0.8352)\end{array}$ \\
\hline Dauer über 12 bis 36 Monate & $\begin{array}{c}-1.0034^{\star *} \\
(0.3099)\end{array}$ & $\begin{array}{r}-1.5142^{*} \\
(0.6436)\end{array}$ & $\begin{array}{l}-4.8699^{\star \star} \\
(0.4798)\end{array}$ & $\begin{array}{c}-9.7013^{\star \star} \\
(0.8351)\end{array}$ \\
\hline Dauer über 36 bis 60 Monate & $\begin{array}{c}-1.1734^{* \star} \\
(0.3148)\end{array}$ & $\begin{array}{c}-1.9441^{\star *} \\
(0.6534)\end{array}$ & $\begin{array}{c}-5.3107^{\star \star} \\
(0.4850)\end{array}$ & $\begin{array}{c}-10.0555^{\star \star} \\
(0.8417)\end{array}$ \\
\hline Dauer über 60 bis 96 Monate & $\begin{array}{c}-1.3716^{\star \star} \\
(0.3188)\end{array}$ & $\begin{array}{c}-2.2642^{\star *} \\
(0.6593)\end{array}$ & $\begin{array}{c}-5.5785^{\star \star} \\
(0.4897)\end{array}$ & $\begin{array}{c}-10.1311^{\star \star} \\
(0.8486)\end{array}$ \\
\hline Dauer über 96 bis 144 Monate & $\begin{array}{c}-1.5939^{\star \star} \\
(0.3231)\end{array}$ & $\begin{array}{c}-2.5299^{\star \star} \\
(0.6688)\end{array}$ & $\begin{array}{c}-5.7136^{\star \star} \\
(0.4930)\end{array}$ & $\begin{array}{c}-10.0092^{\star \star} \\
(0.8519)\end{array}$ \\
\hline Dauer über 144 Monate & $\begin{array}{c}-1.7575^{* *} \\
(0.3203)\end{array}$ & $\begin{array}{c}-2.3901^{\star \star} \\
(0.6628)\end{array}$ & $\begin{array}{c}-6.0386^{\star \star} \\
(0.4934)\end{array}$ & $\begin{array}{c}-10.3008^{* *} \\
(0.8536)\end{array}$ \\
\hline Bildung (pro Schuljahr) & $\begin{array}{c}-0.0409^{\star \star} \\
(0.0078)\end{array}$ & $\begin{array}{c}-0.0290 \\
(0.0169)\end{array}$ & $\begin{array}{l}0.0456^{\star \star} \\
(0.0101)\end{array}$ & $\begin{array}{l}0.0879^{\star \star} \\
(0.0169)\end{array}$ \\
\hline verheiratet ohne Kind & $\begin{array}{l}1.1650^{\star \star} \\
(0.0508)\end{array}$ & $\begin{array}{c}0.4745^{\star \star} \\
(0.1234)\end{array}$ & $\begin{array}{c}-0.5469^{\star \star} \\
(0.0884)\end{array}$ & $\begin{array}{c}-0.0014 \\
(0.1787)\end{array}$ \\
\hline verheiratet mit Kind(ern) & $\begin{array}{c}0.2063^{\star \star} \\
(0.0547)\end{array}$ & $\begin{array}{c}-0.0069 \\
(0.1155)\end{array}$ & $\begin{array}{c}-0.5900^{\star *} \\
(0.0637)\end{array}$ & $\begin{array}{c}0.1128 \\
(0.1168)\end{array}$ \\
\hline Vorschulkind & $\begin{array}{l}0.6133^{* *} \\
(0.0698)\end{array}$ & $\begin{array}{c}0.4631^{\star \star} \\
(0.1458)\end{array}$ & $\begin{array}{c}-0.3298^{\star *} \\
(0.0873)\end{array}$ & $\begin{array}{c}0.0003 \\
(0.1759)\end{array}$ \\
\hline Schulkind & $\begin{array}{l}0.3507^{\star \star} \\
(0.0830)\end{array}$ & $\begin{array}{r}0.3802^{*} \\
(0.1802)\end{array}$ & $\begin{array}{l}0.3131^{\star \star} \\
(0.1004)\end{array}$ & $\begin{array}{c}0.3428 \\
(0.1949)\end{array}$ \\
\hline Kind(er) mindestens 18 & $\begin{array}{l}0.4161^{\star *} \\
(0.1055)\end{array}$ & $\begin{array}{c}0.4866^{\star} \\
(0.2479)\end{array}$ & $\begin{array}{c}0.0841 \\
(0.1614)\end{array}$ & $\begin{array}{c}-0.1031 \\
(0.2723)\end{array}$ \\
\hline Ereignisse & 3570 & 904 & 2167 & 807 \\
\hline
\end{tabular}

Log-likelihood für die simultan geschätzten Übergänge: $-42199,3$ mit d.f. $=30$

** signifikant mit $p<0,01$

* signifikant mit $p<0,05$

Da die Effekte von Alter, Kohorte und Periode (siehe Tabelle 4) im wesentlichen stabil bleiben, wird hier auf deren Wiedergabe verzichtet.

verlassen. Die Eheschließung als solche führt sogar zu einem Anstieg der Übergangsrate um 221 Prozent $((\exp (1,165)-1) \times 100 \%=220,6 \%)$ und macht damit mehr aus, als die beiden Effekte „verheiratet mit Kind(ern)“ und ,.Vorschulkind” zusammengenommen. Aufgrund dieses Ergebnisses könnte man vermuten, daß für traditionell orientierte Frauen die Eheschließung geradezu das auslösende Moment ist, dem Arbeitsmarkt den Rükken zu kehren. Eine andere Erklärung wäre geographische Mobilität bei Heirat, welche oft zu temporärer Erwerbsunterbrechung der Frauen führt. Die Gegenwart von Kindern hat einen geringeren Effekt als Heirat. Die Anwesenheit mindestens eines Kindes im Vorschulalter erhöht das Risiko, die
Vollzeit-Arbeitsstelle zu verlassen, für unverheiratete Mütter um 85 Prozent (Effekt „Vorschulkind“) und für verheiratete Mütter um 127 Prozent (Summe der Effekte „Vorschulkind" und „verheiratet mit Kind(ern)“). Haben alle Kinder das Einschulungsalter erreicht, bedeutet auch dies noch ein erhöhtes Risiko für Erwerbsunterbrechung, und zwar um 75 Prozent für verheiratete bzw. um 42 Prozent für unverheiratete Mütter. In Deutschland erhöht demgegenüber ein Vorschuloder Schulkind die Wahrscheinlichkeit, eine Vollzeiterwerbstätigkeit aufzugeben, um 222 Prozent. Dieser bereits starke Effekt verdoppelt sich noch einmal für verheiratete Mütter (Blossfeld/Rohwer 1995). 
Betrachtet man nun die Erwerbsunterbrechungen bei Teilzeitbeschäftigten, so zeigen sich ähnliche Effekte der Familienereignisse. Sowohl Heirat als auch Kinder erhöhen die Wahrscheinlichkeit für das Verlassen des Beschäftigungssystems. Freilich sind - im Einklang mit den Hypothesen - der Effekt der Heirat sowie der Effekt von Vorschulkindern geringer. Als weiterer wichtiger Unterschied ist festzuhalten, daß ,verheiratet mit Kind(ern)“ im Gegensatz zur Übergangsrate bei Vollzeitbeschäftigten keinen Effekt hat, sich der Familienstand bei teilzeitarbeitenden Müttern also offenbar nicht auf das Verlassen des Arbeitsmarktes auswirkt.

Bei der Wiedereingliederung in den Arbeitsmarkt sind wichtige Unterschiede zwischen der Aufnahme einer Vollzeit- und einer Teilzeitbeschäftigung festzustellen. Unter sonst gleichen Umständen verringert eine Ehe die Wahrscheinlichkeit für die Aufnahme einer Vollzeitbeschäftigung um gut 40 Prozent. Zusätzlich reduziert die Anwesenheit eines Kindes im Vorschulalter die Aufnahme einer Vollzeiterwerbstätigkeit. Wenn sich jedoch alle Kinder im Schulalter befinden, wird die Aufnahme einer Vollzeittätigkeit gefördert. Da der negative Effekt der Ehe aber mitnichten ausgeglichen wird, kann geschlußfolgert werden, daß vor allem unverheiratete Mütter zur Aufnahme einer Vollzeitbeschäftigung neigen, wenn alle Kinder das Schulalter erreicht haben. Wenn schließlich alle Kinder volljährig sind, begünstigt dies die Rückkehr der Mütter auf den Arbeitsmarkt nicht weiter.

Bei den Übergängen in Teilzeitarbeit zeigen sämtliche Familienvariablen wider Erwarten keinerlei Signifikanz. Die Hypothese, nach denen Erwerbsunterbrechungen familienbedingt durch Teilzeitarbeit abgelöst werden, erfährt folglich keine empirische Unterstützung. Die Aufnahme einer Teilzeitbeschäftigung ist nach diesem Ergebnis unabhängig von Veränderungen in den familiären Verpflichtungen nichterwerbstätiger Frauen.

\section{Zusammenfassung und Diskussion}

Die vorgenommene Längsschnittanalyse mit Individualdaten belegt, daß die üblichen Querschnittsanalysen die gestiegene Frauenerwerbstätigkeit nicht hinreichend erklären können. Insbesondere wurde deutlich, daß nicht von weiblichem Erwerbsverhalten ,als solchem“ die Rede sein kann. Erstens ist unter Berücksichtigung familiärer Ereignisse im Kontext des Lebensverlaufes eine Dif- ferenzierung zwischen Arbeitsmarktaus- und -wiedereintritten erforderlich, da der Anstieg der Frauenerwerbstätigkeit in den USA in erster Linie durch verändertes Rückkehrverhalten nach Erwerbsunterbrechungen zu erklären ist. Zweitens ist es wichtig, zwischen Vollzeit- und Teilzeitarbeit zu unterscheiden, da es sich hierbei um strukturell verschiedene Formen der Erwerbsbeteiligung handelt.

In bezug auf Erwerbsunterbrechungen wurden die vermuteten Effekte von Eheschließung und Kindern in der Hauptsache bestätigt, jedoch sind die Effekte familiärer Ereignisse allgemein erheblich schwächer ausgeprägt als in Westdeutschland (Blossfeld/Rohwer 1995). Die Eheschließung ist in den USA immer noch häufig ein Anlaß, die Erwerbstätigkeit - und zwar besonders Vollzeiterwerbstätigkeit - aufzugeben, dies gilt verstärkt für ältere Kohorten. ${ }^{11}$ Die Familienzyklus-Effekte auf Erwerbsunterbrechungen sind bei Vollzeitbeschäftigung etwas stärker als bei Teilzeitbeschäftigung, doch da die Effekte der Heirat und Anwesenheit von Kindern auch bei Teilzeitbeschäftigten vorhanden sind, muß die Hypothese einer optimalen Vereinbarkeit von Kindererziehung und Teilzeitarbeit abgeschwächt werden.

Lassen sich Erwerbsunterbrechungen somit in der Längsschnittperspektive auf das Merkmal Lebensalter und auf familiäre Ereignisse zurückführen, so ist die Sachlage im Hinblick auf Wiedereintritte in das Beschäftigungssystem komplexer. In Deutschland, wo Teilzeitarbeit in die soziale Sicherung eingebunden ist und das Steuersystem nicht gerade Anreize für eine Vollzeiterwerbstätigkeit beider Ehepartner setzt, ist Teilzeitarbeit die wichtigste Form der Erwerbstätigkeit bei der Rückkehr der Hausfrau in das formelle Beschäftigungssystem (Pfau-Effinger 1994; Schupp 1991; Blossfeld/Rohwer 1995).

Für amerikanische Frauen wirken sich familiäre Ereignisse auf die Aufnahme einer Vollzeitbeschäftigung aus. Es ergaben sich aber nicht die erwarteten Effekte vom Alter der Kinder auf Übergänge von Nichterwerbstätigkeit in Teilzeitarbeit. Dieses Ergebnis ist insofern unerwartet, als daß im allgemeinen zumindest stillschweigend angenommen wird, daß amerikanische Frauen in Teilzeitarbeit niedrige Bezahlung, schlechte Aufstiegschancen und soziale Absicherung in Kauf nehmen, um

1 Hier nicht wiedergegebene Schätzungen von Modellen mit Interaktionseffekten zwischen Kohorte und Heirat haben gezeigt, daß der Effekt der Heirat für ältere Kohorten bedeutend stärker ist als für jüngere. 
eine bessere Vereinbarkeit von Familie und Beruf zu erreichen. Unsere Analyse zeigt, daß nicht familiäre Phasen, sondern vielmehr jüngeres Lebensalter, höhere Bildung und besonders Zugehörigkeit zu einer jüngeren Geburtskohorte diejenigen Faktoren sind, welche die Wahrscheinlichkeit erhöhen, aus einer Phase der Nichterwerbstätigkeit heraus teilzeit zu arbeiten. Aus diesen Gründen ist es fraglich, ob Vorschläge - wie von Moen/ Dempster-McClain (1987) - Familie und Beruf in den USA durch mehr Teilzeitarbeit miteinander auszusöhnen, erfolgversprechend sein können. ${ }^{12}$ Andere Autoren behaupten, daß vermehrt Teilzeitarbeitsplätze geschaffen werden, weil sie den Arbeitgebern als Mittel zur Dämpfung von Arbeitskosten dienen und nicht weil die Präferenz für Teilzeitarbeit zunimmt (Tilly 1991).

In Westdeutschland ist Teilzeitarbeit besser in das soziale Sicherungssystem eingebunden und das Arbeitsmarktverhalten der Frauen hängt deutlich von familiären Ereignissen ab. In den USA sind Teilzeitstellen hinsichtlich sozialer Leistungen viel schlechter ausgestattet als Vollzeitstellen. Besonders schwerwiegend ist, daß Teilzeitkräfte kaum unter den Schutz der Krankenversicherung fallen, was aber besonders für nicht verheiratete Mütter, die finanziell schlecht gestellt sind, ein entscheidender Punkt ist. Harris (1993) hat darauf hingewiesen, daß sich alleinerziehende Frauen eine Teilzeitarbeit ohne Krankenversicherung schlichtweg nicht leisten können. Sie stehen vor der Alternative, entweder ohne Arbeit von Sozialhilfe, welche medizinische Betreuung einschließt, zu leben oder aber cine Vollzeitstelle mit ausreichender Bezahlung und sozialer Sicherung zu finden.

Ein weiteres wichtiges Ergebnis unserer Analyse ist, daß das zentrale Argument von Goldin (1990), daß Kohorten Träger des sozialen Wandels der Frauenerwerbstätigkeit seien, revidiert werden muß. Sie kam zu ihrer Schlußfolgerung, ohne zwischen Vollzeit- und Teilzeitarbeit unterschieden zu haben. Unsere Analyse zeigt, daß die Zunahme der Vollzeitbeschäftigung in den USA seit den 70er Jahren alle Frauen betrifft. Lediglich Teilzeitarbeit kann als Kohortenphänomen aufgefaßt werden.

${ }^{12}$ Ebenso ist es fraglich, ob die Politik einer generellen Umverteilung von Arbeit durch Teilzeitarbeit in Deutschland erfolgreich sein kann, wenn man deren Abhängigkeit von spezifischen Familienphasen im weiblichen Lebenslauf in Rechnung stellt.

\section{Literatur}

Allison, P.D., 1984: Event History Analysis. Regression for Longitudinal Event Data. SAGE series: Quantitative Applications in the Social Sciences.

Becker, G.S., 1981: A Treatise on the Family. Cambridge: Harvard University Press.

Beechey, V./Perkins, T., 1987: A Matter of Hours. Women, Part-time Work and the Labour Market. Minneapolis: University of Minnesota Press.

Bernhardt, E.M., 1993: Fertility and Employment. European Sociological Review 9(1): 25-42.

Blank, R.M., 1989: The Role of Part-Time Work in Women's Labor Market Choices Over Time. The American Economic Review 79(2): 295-299.

Blossfeld, H.-P., 1995: Family Cycle and Growth in Women's Part-time Employment in Western European Countries. In: H.-P. Blossfeld (Hrsg.), 1995: Between Equalization and Marginalization. Part-time Working Women in Europe and the United States. Oxford (erscheint demnächst).

Blossfeld. H.-P./Hamerle, A./Mayer, K.U., 1989: Event History Analysis. Statistical Theory and Application in the Social Sciences. Hillsdale, NJ: Lawrence Erlbaum Associates.

Blossfeld, H.-P./Rohwer, G., 1995: Family Cycle and Growth in Women's Part-time Work in West-Germany. In: H.-P. Blossfeld (Hrsg.), 1995: Between Equalization and Marginalization. Part-time Working Women in Europe and the United States. Oxford (erscheint demnächst).

Bumpass, L.L., 1990: What's Happening to the Family? Interactions Between Demographic and Institutional Change. Demography 27: 483-498.

Burchell, B./Dale, A./Joshi, H., 1993: Part-time Working amongst British Women. In: H.-P. Blossfeld (Hrsg.), 1995: Between Equalization and Marginalization. Parttime Working Women in Europe and the United States. Oxford (erscheint demnächst).

Burt, R.S., 1991: Measuring Age as a Structural Concept. Social Networks 13: 1-34.

Cramer, J.C., 1980: Fertility and Female Employment: Problems of Causal Direction. American Sociological Review 45: 167-190.

DaVanzo, J./Rahman, M.O., 1993: American Families: Trends and Correlates. Population Index 59(3): 350386.

Drobnič, S., 1995: Part-time Work in Central and East European Countries. In: H.P. Blossfeld (Hrsg.): Between Equalization and Marginalization. Part-time Working Women in Europe and the United States. Oxford (erscheint demnächst).

Economic Report of the President, 1992. Washington: United States Government Printing Office.

Ellingsater, A.L.. 1992: Part-time Work in European Welfare States. Denmark. Germany, Norway and the United Kingdom Compared. Oslo: ISF.

Gerson, K.. 1985: Hard Choices. How Women Decide about Work, Career, and Motherhood. Berkeley/Los Angeles: University of California Press. 
Glick, P.C., 1977: Updating the Life Cycle of the Family. Journal of Marriage and the Family 39(1): 5-13.

Goldin, C., 1990: Understanding the Gender Gap. An Economic History of American Women. New York/Oxford: Oxford University Press.

Hakim, C., 1993: The Myth of Rising Female Employment. Work, Employment \& Society 7(1): 97-120.

Handl, J., 1988: Berufschancen und Heiratsmuster von Frauen. Empirische Untersuchungen zu Prozessen sozialer Mobilität. Frankfurt/New York: Campus.

Harris, K.M., 1993: Work and Welfare among Single Mothers. American Journal of Sociology 99(2): 317-352.

Hesse, S.J., 1979: Women Working: Historical Trends. S. 35-62 in: K.W. Feinstein (Hrsg.): Working Women and Families. Beverly Hills/London: Sage.

Hobcraft, J./Menken,J./Preston, S., 1982: Age. Period, and Cohort Effects in Demography: A Review. Population Index 48(1): 4-43.

ILO, 1989: Conditions of Work Digest 8(1). Geneva.

Jacobs, J.A., 1989: Revolving Doors. Sex Segregation and Women's Careers. Stanford, CA: Stanford University Press.

Kalbfleisch, J.D./Prentice, R.L.. 1980): The Statistical Analysis of Failure Time Data. New York: Wiley.

Kalleberg, A.L./Rosenfeld, R.A., 1990: Work in the Family and in the Labor Market: A Cross-national, Reciprocal Analysis. Journal of Marriage and the Family 52: 331346.

Masnick, G./Bane, M.J., 1980: The Nation's Families: 19601990. Boston: Auburn Housc.

Mellor, E.F./Parks Il, W., 1988: A Year's Work: Labor Force Activity from a different Perspective. Monthly Labor Review 111(9): 13-18.

Mincer, J./Ofek, H., 1982: Interrupted Work Careers: Depreciation and Restoration of Human Capital. The Journal of Human Resources 12: 3-24.

Mincer, J./Polachek, S.W., 1974: Family Investments in Human Capital: Earnings of Women. Journal of Political Economy 82: 76-108.

Moen, P./Dempster-McClain, D.I., 1987: Employed Parents: Role Strain, Work Time, and Preferences for Working Less. Journal of Marriage and the Family 49: 579-590.

Moen, P./Smith, K.R., 1986: Women at Work: Commitment and Bchavior Over the Life Course. Sociological Forum 1(3): $450-475$

Müller, W., 1983: Frauenerwerbstätigkeit im Lebenslauf. S. 55-106 in: Müller. W./Willms, A./Handl, J.: Strukturwandel der Frauenarbeit 1880-1980. Frankfurt/New York: Campus.

Murphy, M.. 1991: The Collection and Comparability of Demographic and Social Data in Europe. Chaire Quetelet Seminar, 17-20 September. Gembloux.

NSFH (A National Survey of Families and Houscholds). 1988: Codebook and Documentation. Center for Demography and Ecology. University of Wisconsin, Madison.

Oppenheimer, V.K., 1973: Demographic Influence on Female Employment and the Status of Women. American Journal of Sociology 78(4): 946-961.
Oppenheimer. V.K., 1974: The Life-Cycle Squeeze: The Interaction of Men's Occupational and Family Life Cycles. Demography 11 (2): 227-245.

Pfau-Effinger, B., 1994: Erwerbspartnerin oder berufstätige Ehefrau. Sozio-kulturelle Arrangements der Erwerbstätigkeit von Frauen im Vergleich. Soziale Welt 45(3): 322-337.

Polachek, S.W., 1981: Occupational Self-Selection: A Human Capital Approach to Sex Differences in Occupational Structure. Review of Economics and Statistics 63 $60-69$.

Popenoe, D., 1993: American Family Decline, 1960-1990: A Review and Appraisal. Journal of Marriage and the Family 55: 527-555.

Presser, H.B., 1994: Employment Schedules among DualEarner Spouses and the Division of Household Labor by Gender. American Sociological Review 59: 348-364.

Quack, S., 1993: Dynamik der Teilzeitarbeit. Implikationen für die soziale Sicherung von Frauen. Berlin: Ed. Sigma.

Riley. M.W.. 1987: On the Significance of Age in Sociology. American Sociological Review 52: 1-14.

Rohwer, G., 1994: TDA Working Papers.

Ryder. N.B., 1985 [1965]: The Cohort as a Concept in the Study of Social Change. S. 9-44 in: W.M. Mason/S.E. Fienberg (Hrsg.): Cohort Analysis in Social Research. New York/Berlin/Heidelberg/Tokyo: Springer.

Schupp, J.. 1991: Teilzeitarbeit als Möglichkeit der beruflichen (Re-)Integration. Empirische Analysen auf der Basis aktueller Längsschnittdaten. S. 207-232 in: K.U. Mayer/J. Allmendinger/J. Huinink (Hrsg.): Vom Regen in die Traufe: Frauen zwischen Beruf und Familie. Frankfurt/New York: Campus.

Smith, J.P./Ward, M.. 1989: Women in the Labor Market and in the Family. Journal of Economic Perspectives 3(1): 9-23.

Statistical Abstract of the United States. Diverse Ausgaben.

Stromberg, A.H./Harkess, S., 1978: Editor's Introduction. S. 3.9 in: A.H. Stromberg/S. Harkess (Hrsg.): Women Working. Theories and Facts in Perspective. Mayfield Publishing Company.

Sundström. M., 1991: Part-Time Work in Sweden: Trends and Equality Effects. Journal of Economic Issues 25(1): $167-178$.

Teachman, J.D./Polonko, K.A./Scanzoni, J., 1987: Demography of the Family. S 3-36 in: M.B. Sussman/S.K. Steinmetz (Hrsg.): Handbook of Marriage and the Family. New York/London: Plenum Press.

Tilly, C., 1991: Reasons for the Continuing Growth of Parttime Employment. Monthly Labor Review 114(3): 1018.

Tuma, N.B./Hannan, M.T., 1984: Social Dynamics. Models and Methods. Academic Press.

Waitc. L.J., 1981: U.S. Women at Work. Population Bulletin 36(2). Washington. D.C.: Population Reference Bureau.

Wandersee, W.D., 1981: Women's Work and Family Values 1920-1940. Cambridge, MA/London: Harvard Univcrsity Press.

Willms-Herget. A., 1985: Frauenarbeit. Zur Integration der Frauen in den Arbeitsmarkt. Frankfurt/New York: Campus. 